\title{
Electromagnetic Interference and Safety in Wireless Networks: A Statistical Approach
}

\author{
Sergey Loyka \\ School of Information Technology and Engineering \\ University of Ottawa \\ 161 Louis Pasteur, Ottawa, Canada, K1N 6N5 \\ E-mail: sergey.loyka@ieee.org
}

\author{
Vladimir Mordachev \\ Electromagnetic Compatibility Laboratory \\ Belorussian State University of Informatics and \\ Radioelectronics (BSUIR) \\ 6 P.Brovki st., Minsk 220013, Belarus \\ E-mail: emc@bsuir.by
}

\begin{abstract}
Statistical properties of electromagnetic environment in wireless networks affecting its performance and safety are studied. A statistical method to evaluate risks to the general public due to electromagnetic radiation in wireless networks as well as their performance is proposed. The analysis is based on the standard propagation channel model, a Poisson model of random spatial distribution of transmitters, and a threshold-based model of the victim receptor behaviour (radio receiver or human body). The distribution of dominant interference level is derived and analysed under various network and system configurations. The aggregate interference is dominated by the nearest one. The outage probability is used as a measure of not only the wireless link quality-of-service, but also of environmental risks induced by electromagnetic radiation. The maximum acceptable interference levels for reliable link performance and for low environmental risks are shown to be surprisingly similar.
\end{abstract}

\section{INTRODUCTION}

Performance and safety of wireless communication networks have been recently a subject of extensive studies. Mutual interference among several links (e.g. several users) operating at the same time places a fundamental limit to the network performance and also determines the level of electromagnetic environmental risks to the general public. While wireless communications experience an era of unprecedented growth and deliver a number of important services, there is also a negative side of it in the form of electromagnetic "pollution" of the environment. A traditional method of control of this pollution is via the maximum permissible levels of radiation (exposure) [7]. While this approach is able to control the radiation of most powerful emitters (e.g. base stations of cellular systems), it is not able to control the radiation of a large number of relatively lowpower devices (e.g. cell phones), since their individual power is well below the established radiation limits. Limiting highpower exposure is able to eliminate short-term health risks (e.g. due to termal effects), which are deterministic in nature and exhibit a threshold effect. However, there remains longterm effects, which are due to low exposure levels (over an extended period of time) and which are probabilistic in nature and also without a threshold effect [9]. There has been recently growing evidence that low-level radiation of cell phones is able to stimulate serious negative effects (e.g. cancer) when applied for a long period of time [10]. These long-term effects call for a new method of estimating and controlling electromagnetic environmental risks, which is able to account for aggregate radiation levels of many lowpower sources (e.g. cell phones, wireless Internet access devices, local area and body-area networks etc.). Such a method is proposed here, which is based on a well-developed statistical approach to analyze interference effects in wireless communication networks [1]-[6].

The total power $P_{T}$ absorbed by a receptor includes two components a static (deterministic) power $P_{s}$ from fixed high-power emitters at fixed locations (e.g. base stations) and highly-variable random power $P$ from mobile devices,

$$
P_{T}=P_{s}+P,
$$

While evaluation of the deterministic component $P_{S}$ is well -documented [8] (either via computation or measurement), the random component $P$ is not well-understood and a method to evaluate it is proposed here via the concept of outage probability, i.e. the probability that random interference level exceeds a given threshold. In many cases the radiation of nearby cell phones significantly exceeds that of a far-away base station so that the former is dominant (geographically speaking), $P_{s} \ll P$ and therefore $P_{T} P$. We remark that statistical methods of evaluation of radiation exposure of the general public were used before in different contexts [9] (e.g. X-ray) but, to the best of our knowledge, were never applied to the context of wireless networks and their environmental risks.

The effect of interference in wireless networks at the physical layer of communication systems/networks has been studied from several perspectives [1]-[6]. A typical statistical model of electromagnetic interference in a wireless network includes a model of spatial location of the nodes (transmitters), a propagation path loss law (which includes the average path loss and, possibly, large-scale (shadowing) and small-scale (multipath) fading) and a threshold-based receiver (receptor) performance model. The most popular choice for the model of the node spatial distribution is Poisson point process on a plane [1]-[2]. A common feature of all these works is the use of aggregate interference (either alone or in the form of signal-to-interference-plus-noise ratio), and a common lesson is that it is very difficult to deal with: while the characteristic function $(\mathrm{CF})$ of aggregate interference can be obtained in a closed form, the probability density function (PDF) or cumulative distribution function (CDF) are available only in a few special cases. This limits significantly the amount of insight that can be extracted from such a model, especially if no approximations or bounds are 
used.

To overcome this difficulty, we adopt a different approach: instead of relying on the aggregate interference power as a performance indicator, we use the power of the dominating interfering signal [4]-[6]. While this is clearly an approximation, closed-form performance evaluation becomes feasible and significant insight can be extracted from such a model. Furthermore, since the aggregate interference is dominated by the most powerful interferer in the region of low outage probability (i.e. the practicallyimportant region), both models give roughly the same results (see [6] for details).

Using this model, we study the power distribution of the ensemble of interferences and the dominant interferer in various scenarios, which is further used to obtain compact closed-form expressions for the outage probability of a given receptor (or, equivalently, of the link of a given user) in the 1-D, 2-D and 3-D Poisson field of interferers, for both uniform and non-uniform average node densities and for various values of the average path loss exponent. The proposed method is flexible enough to include the case when a given number of nearest interferers are not active (e.g. due to the MAC protocol). The outage probability is shown to scale down exponentially in this number. The proposed method can also be used to include the effect of fading. We argue that Rayleigh fading has a negligible effect on the distribution of dominant interferer's power and the effect of log-normal fading (shadowing) is to shift the distribution by a constant non-negligible factor [6].

Our analysis culminates in the formulation of the outage probability-network density tradeoff: for a given average density of the nodes, the outage probability is lower bounded or, equivalently, for a given outage probability, the average density of the nodes is upper bounded. This tradeoff is a result of the interplay between a random geometry of node locations, the propagation path loss and the distortion effects at the victim receiver.

We argue that the outage probability, which is traditionally used as a measure of quality-of-service in wireless systems and networks, also measures the environmental risks to the population induced by electromagnetic radiation of wireless devices.

The paper is organized as follows. In Section 2, we introduce the system and network model. In Section 3, the distribution of interference levels and of the dynamic range (dominant interference-to-noise ratio) is given for this model. Based on this, the node density - outage probability tradeoff is presented in Section 4. The maximum acceptable interference levels for high quality-of-service wireless network performance and for low electromagnetic environmental risks to the general public are shown to be surprisingly similar in Section 5.

\section{NETWORK AND SYSTEM MODEL}

As an interference model of wireless network at the physical layer, we consider a number of point-like transmitters $(\mathrm{Tx})$ and receptors $(\mathrm{Rx})$ that are randomly located over a certain limited region of space $S_{m}$, which can be one $(m=1)$, two $(m=2)$, or three $(m=3)$-dimensional (1-D, 2-D or 3-D). This can model location of the nodes over a highway or a street canyon (1-D), a residential area (2-D), or a downtown area with a number of high-rise buildings (3D). In our analysis, we consider a single (randomly-chosen) receiver (or some other receptor which is susceptible to electromagnetic fields generated by transmitters) and a number of transmitters that generate interference to this receiver. We assume that the spatial distribution of the transmitters (nodes) has the following properties: (i) for any two non-overlapping regions of space $S_{a}$ and $S_{b}$, the probability of any number of transmitters falling into $S_{a}$ is independent of how many transmitters fall into $S_{b}$, i.e. nonoverlapping regions of space are statistically independent; (ii) for infinitesimally small region of space $d S$, the probability $\mathcal{P}(k=1, d S)$ of a single transmitter $(k=1)$ falling into $d S$ is $\mathcal{P}(k=1, d S)=d S$, where is the average spatial density of transmitters (which can be a function of position). The probability of more than one transmitter falling into $d S$ is negligible, $\mathcal{P}(k>1, d S)<<\mathcal{P}(k=1, d S) \quad$ as $\quad d S \quad 0$. Under these assumptions, the probability of exactly $k$ transmitters falling into the region $S$ is given by Poisson distribution,

$$
\mathcal{P}(k, S)=e^{\bar{N}} \bar{N}^{k} / k !, \bar{N}=\int_{S} d S
$$

where $\bar{N}$ is the average number of transmitters falling into the region $S$. If the density is constant, then $\bar{N}=S$. Possible scenarios to which the assumptions above apply, with a certain degree of approximation, are a sensor network with randomly-located non-cooperating sensors; a network(s) of mobile phones from the same or different providers (in the same area); a network of multi-standard wireless devices sharing the same resources (e.g. common or adjacent bands of frequencies) or an ad-hoc network.

Consider now a given transmitter-receptor (transmitterreceiver) pair. The power at the $\mathrm{Rx}$ antenna output $P_{r}$ coming from the transmitter is given by the standard link budget equation [3],

$$
P_{r}=P_{t} G_{t} G_{r} g
$$

where $P_{t}$ is the Tx power, $G_{t}, G_{r}$ are the Tx and Rx antenna gains, and $g$ is the propagation path gain ( $=1 /$ path loss), $g=g_{a} g_{l} g_{s}$, where $g_{a}$ is the average propagation path gain, and $g_{l}, g_{s}$ are the contributions of large-scale (shadowing) and small-scale (multipath) fading, which can be modeled as independent log-normal and Rayleigh (Rice) random variables, respectively [3].

The widely-accepted model for $g_{a}$ is $g_{a}=a R$, where

is the path loss exponent, and $a$ is constant independent of $R$ [3]. In the traditional link-budget analysis of a pointto-point link, it is a deterministic constant. However, in our network-level model $g_{a}$ becomes a random variable since the Tx-Rx distance $R$ is random (due to random location of the nodes) and it is this random variable that represents a new type of fading, which we term "network-scale fading", since it exhibits itself on the scale of the whole area occupied by the network. Since $g_{a}$ does not depend on the local propagation environment around the $\mathrm{Tx}$ or $\mathrm{Rx}$ ends that affect $g_{l}, g_{s}$ but only on the global configuration of the Tx$\mathrm{Rx}$ propagation path (including the distance $R$, of which $g_{l}, g_{s}$ are independent) [3], the network-scale fading in this model is independent of the large-scale and small-scale ones, 
which is ultimately due to different physical mechanisms generating them. The distribution functions of $g_{a}$ in various scenarios have been given in [4]-[5].

\section{CDF OF INTERFERENCE AND THE INTERFERENCE TO NOISE RATIO}

We consider a fixed-position receptor and a number of randomly located interfering transmitters (interferers, e.g. mobile units of other users) of the same power $P_{t}$ (following the framework in [4]-[6], this can also be generalized to the case of unequal Tx powers). Only the network-scale fading is taken into account in this section, assuming that $g_{l}=g_{s}=1$ (this assumption is relaxed in section 4). For simplicity, we also assume that the Tx and Rx antennas are isotropic (this assumption is relaxed below), and consider the interfering signals at the receiver input.

The statistics of transmitters' location is given by (2). Transmitter $i$ produces the average power $P_{a i}=P_{t} g_{a}\left(R_{i}\right)$ at the receiver input, and we consider only the signals exceeding the Rx noise level $P_{0}, P_{a i} P_{0}$. We define the interference-to-noise ratio (INR) $d_{a}$ in the ensemble of the interfering signals via the most powerful (at the Rx input) signal (It can be shown that, in the small outage region, the total interference power (i.e. coming from all transmitters) is dominated by the contribution of the most powerful signal, i.e. the single events dominate the outage probability [6]),

$$
d_{a}=P_{a 1} / P_{0}
$$

where, without loss of generality, we index the transmitters

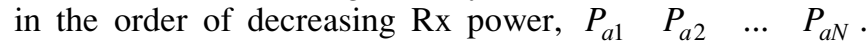
The most powerful signal is coming from the transmitter located at the minimum distance $r_{1}, P_{a 1}=P_{t} g_{a}\left(r_{1}\right)$. The cumulative distribution function (CDF) of the minimum distance can be easily found [4]-[6],

$$
F_{1}(r)=1 \quad \exp (\bar{N}(V)), \bar{N}(V)=\int_{V} d V,
$$

where $\bar{N}(V)$ is the average number of transmitters in the ball $V(r)$ of radius $r$. The probability that the INR exceeds value $D$ is $\operatorname{Pr}\left\{d_{a}>D\right\}=\operatorname{Pr}\left\{r_{1}<r(D)\right\}=F_{1}(r(D))$, where $r(D)$ is such that $P_{a}(r(D))=P_{0} D$, so that the CDF of $d_{a}$ is

$$
F_{d}(D)=1 \quad \operatorname{Pr}\left\{d_{a}>D\right\}=\exp (\bar{N}(D))
$$

where $\bar{N}(D)$ is the average number of transmitters in the ball $V(r(D))$ of the radius $r(D)=\left(P_{t} a / P_{0} D\right)^{1 /}$. When the average spatial density of transmitters is constant, = const, (6) simplifies to [5][6],

$$
F_{d}(D)=\exp \left\{c_{m}\left(\frac{P_{t} a}{P_{0} D}\right)^{m /}\right\}=\exp \left\{\bar{N}_{\max } D^{m /}\right\},
$$

where $c_{1}=2, c_{2}=$ and $c_{3}=4 / 3, \bar{N}_{\max }=c_{m} R_{\max }^{m}$ is the average number of transmitters in the ball of radius $R_{\max }$, which we term "potential interference zone", and $R_{\max }$ is such that $P_{a}\left(R_{\max }\right)=P_{0}$, i.e. a transmitter at the boundary of the potential interference zone produces signal at the receiver exactly at the noise level; transmitters located outside of this zone produce weaker signals, which are neglected in the analysis. Note that (7) gives the distribution of the INR as a simple explicit function of the system and geometrical parameters, and ultimately depends on $N_{\text {max }}, m$, only.

When $(k 1)$ most powerful signals, which are coming from $(k 1)$ closest transmitters, do not create any interference (i.e. due to frequency, time or code separation in the multiple access scheme, or due MAC protocol not allowing them to transmit), the CDF of the distance $r_{k}$ to the most powerful interfering signal of order $k$ can be found in a similar way. The CDF of the INR $d_{a}$ in this case is given by

$$
F_{d k}(D)=e^{\bar{N}(D)} \sum_{i=0}^{k} \bar{N}(D)^{i} / i !
$$

In the case of constant average density = const, the $\mathrm{CDF}$ of the INR simplifies to [5][6],

$$
F_{d k}(D)=\exp \left\{\bar{N}_{\max } D^{m /}\right\} \sum_{i=0}^{k} \frac{1}{i !}\left(\frac{\bar{N}_{\max }}{D^{m /}}\right)^{i},
$$

which are also explicit functions of $\bar{N}_{\max }, m$, .

\section{Outage PRobability-Node Density TradeOFF}

Powerful interfering signals can result in significant performance degradation due to linear and nonlinear distortion effects in the receiver when they exceed certain limit, which we characterize here via the receiver distortionfree dynamic range (i.e. the maximum acceptable interference-to-nose ratio) $D_{d f}=P_{\max } / P_{0}$, where $P_{\max }$ is the maximum interfering signal power at the receiver that does not cause significant performance degradation. If $d_{a}>D_{d f}$, there is significant performance degradation and the receiver is considered to be in outage, which corresponds to one or more transmitters falling into the active interference zone (i.e. the ball of radius $r\left(D_{d f}\right)$; the signal power coming from transmitters at that zone exceeds $P_{\max }$ ), whose probability is

$$
\mathcal{P}_{\text {out }}=\operatorname{Pr}\left\{d_{a}>D_{d f}\right\}=1 \quad F_{d}\left(D_{d f}\right)
$$

For given $\mathcal{P}_{\text {out }}$, one can find the required distortion-free dynamic range ("outage dynamic range") $D_{d f}$

$$
D_{d f}=F_{d}{ }^{1}\left(1 \quad \mathcal{P}_{\text {out }}\right)
$$

We note that, in general, $D_{d f}$ is a decreasing function of $\mathcal{P}_{\text {out }}$, i.e. low outage probability calls for high distortion-free dynamic range. For simplicity of notations, we further drop the subscript and denote the spurious-free dynamic range by $D$.

All interfering signals are active $(k=1)$ : We consider first the case of $k=1$, i.e. all interfering signals are active. The outage probability can be evaluated using (6) and (10). From practical perspective, we are interested in the range of small outage probabilities $\mathcal{P}_{\text {out }}<<1$, i.e. high-reliability and communications. When this is the case, $F_{d}(D)-1$ and using MacLaurean series expansion $e^{\bar{N} d} 1 \bar{N}$, (10) simplifies to

$$
\mathcal{P}_{\text {out }} \quad \bar{N}=\int_{V(r(D))} d V
$$

which further simplifies, in the case of $=$ const, to

$$
\mathcal{P}_{\text {out }} \quad \bar{N}_{\max } D^{m /}
$$

Note that, in this case, the outage probability $\mathcal{P}_{\text {out }}$ scales 
linearly with the average number $\bar{N}_{\max }$ of nodes in the potential interference zone, and it effectively behaves as if the number of nodes were fixed (not random) and equal to $\bar{N}_{\max }$. Based on this, we conclude that the single-order events (i.e. when only one signal in the ensemble of interfering signals exceeds the threshold $P_{\max }$ ) are dominant contributor to the outage. This immediately suggests a way to reduce significantly the outage probability by eliminating (e.g. by filtering) the dominant interferer in the ensemble. Using (13), the required spurious-free dynamic range of the receiver can be found for given outage probability, $D \quad\left(\bar{N}_{\max } / \mathcal{P}_{\text {out }}\right)^{/ m}$. Note that higher values of and lower values for $m$ call for higher dynamic range. Intuitively, this can be explained by the fact that when the transmitter moves from the boundary of the potential interference zone (i.e. $\left.R=R_{\max }, P_{a}(R)=P_{0}\right)$ closer to the receiver $\left(R<R_{\max }\right)$, the power grows much faster when is larger, so that closely-located transmitters produce much more interference (compared to those located close to the boundary) when is large, which, combined with the uniform spatial density of the transmitters, explains the observed behavior. The effect of $m$ can be explained in a similar way.

To validate the accuracy of approximation in (12), and also the expressions for the dynamic range PDF and CDF in the previous section, extensive Monte-Carlo (MC) simulations have been undertaken. Fig. 1 shows some of the representative results. Note good agreement between the analytical results (including the approximations) and the MC simulations. It can be also observed that the tails of the distributions decay much slower for the $=4$ case, which indicates higher probability of high-power interference in that case and, consequently, requires higher spurious-free dynamic range of the receiver, in complete agreement with the predictions of the analysis. Note also that the outage probability evaluated via the total interference power coincides with that evaluated via the maximum interferer power, at the small outage region (this result has been rigorously proved in [6]).

Consider now a scenario where the actual outage probability has not to exceed a given value $\mathcal{P}_{\text {out }}$ for the receiver with a given distortion-free dynamic range $D$. Using (7) and (10), the average number of transmitters in the active interference zone (ball of radius $r(D)$ ) can be upper bounded as $\bar{N} \quad \ln \left(1 \mathcal{P}_{\text {out }}\right)$. Using the expression for $\bar{N}$, one obtains a basic tradeoff relationship between the network density and the outage probability,

$$
\bar{N}=\int_{V(r(D))} d V \quad \ln \left(1 \quad \mathcal{P}_{\text {out }}\right)
$$

i.e. for given outage probability, the network density is upper bounded or, equivalently, for given network density, the outage probability is lower bounded.

In the case of uniform density = const and small outage probability, $\mathcal{P}_{\text {out }}<<1$, this gives an explicit tradeoff relationship between the maximum distortion-free interference power at the receiver $P_{\max }$, the transmitter power $P_{t}$ and the average node density for distortion-free receiver operation,

$$
c_{m}{ }^{1} \mathcal{P}_{\text {out }}\left(P_{\max } / P_{t} a\right)^{m /}
$$

or, equivalently, an upper bound on the average density of nodes in the network. As intuitively expected, higher $\mathcal{P}_{\text {out }}, P_{\max }$, and lower $P_{t}, m$ allow for higher network density. The effect of is intuitively explained by the fact that higher results in larger path loss or, equivalently, in smaller distance at the same path loss, so that the transmitters can be located more densely without increasing interference level. The effect of the other parameters can be explained in a similar way.

$\left(\begin{array}{ll}k & 1\end{array}\right)$ strongest interfering signals are inactive: We now assume that $\left(\begin{array}{lll}k & 1\end{array}\right)$ strongest interfering signals are eliminated via some means (e.g. by filtering or resource allocation). In this case, (8), (9) apply and (12) generalizes to

$$
\mathcal{P}_{\text {out }} \frac{1}{k !} \bar{N}^{k}=\frac{1}{k !}\left(\bar{N}_{\max } D^{m /}\right)^{k}
$$

which can be expressed as $\mathcal{P}_{\text {out }}=\frac{1}{k !} \mathcal{P}_{\text {out }, 1}^{k} \mathcal{P}_{\text {out }, 1}$, where $\mathcal{P}_{\text {out }, 1}$ is the outage probability for $k=1$ (see (12)). In the small outage region, $\mathcal{P}_{\text {out }, 1}<<1$ and $\mathcal{P}_{\text {out }}<<\mathcal{P}_{\text {out }, 1}$, i.e. there is a significant beneficial effect of removing $(k 1)$ strongest interferers, which scales exponentially with $k$. It should also be noted that, contrary to the $k=1$ case, $\mathcal{P}_{\text {out }}$ in (16) is super-linear in $\bar{N}_{\text {max }}$ : doubling $\bar{N}_{\text {max }}$ increases $\mathcal{P}_{\text {out }}$ by the factor $2^{k}>2$, i.e. $\mathcal{P}_{\text {out }}$ is more sensitive to $\bar{N}_{\max }$ in this case.

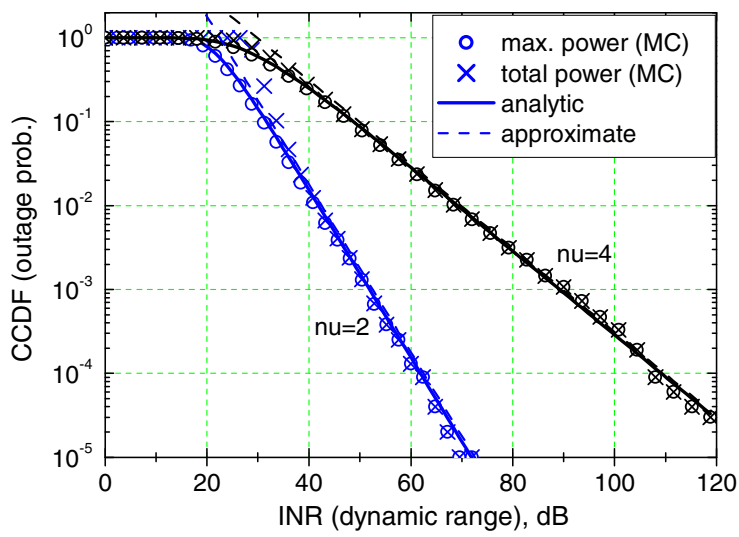

Fig. 1. The CCDF of $d_{a}=P_{a 1} / P_{0}$ and $d_{t o t}=P_{t o t} / P_{0}$ (also the outage probability) evaluated from Monte-Carlo (MC) simulations for $m=2, \quad=2 \& 4, P_{0}=10^{10}, P_{t}=1, \quad=10^{5}$; analytic CCDF of $d_{a}$ (derived from (7)) and its approximation in (13) are also shown. Note significant dependence of outage probability on the propagation conditions.

In a similar way, the node density-outage probability tradeoff can be formulated. In the for small outage probability region $\mathcal{P}_{\text {out }}<<1$, it can be expressed as

$$
\bar{N}=\int_{V(r(D))} d V \quad\left(k ! \mathcal{P}_{\text {out }}\right)^{1 / k}
$$

Comparing (17) to (14), one can clearly see the beneficial effect of "removing" $\left(\begin{array}{ll}k & 1\end{array}\right)$ most powerful interferers on the outage probability-network density tradeoff, since $\left(k ! \mathcal{P}_{\text {out }}\right)^{1{ }^{\varphi k}} \gg \mathcal{P}_{\text {out }}$ in the small outage regime, so that higher node density is allowed at the same outage probability.

In the case of uniform density, (17) reduces to 


$$
c_{m}{ }^{1}\left(k ! \mathcal{P}_{\text {out }}\right)^{1 / k}\left(P_{\max } / P_{t} a\right)^{m /}
$$

which is a generalization of (15) to $k 1$.

Impact of Rayleigh and log-normal fading: Following the approach presented in [6], it can be shown that the impact of Rayleigh and log-normal fading on the distributions above is a shift by a constant factor. In the case of Rayleigh fading, the constant is close to 1 and, thus, can be neglected so that the distributions are roughly not affected. In the case of lognormal fading, the constant is not negligible. The intuition behind this result is that the distributions in (10), (13), (16) are much more heavily-tailed (slowly-decaying) than the Rayleigh distribution so that outage events in the combined distribution are mostly caused by nearby interferers without deep Rayleigh fades and the combined distribution is roughly the same as the original one (without fading). On the other hand, the log-normal distribution is also heavily-tailed, so it cannot be neglected (see [6] for a detailed analysis of the fading effects).

\section{Outage Probability as A Statistical Measure of ELECTROMAGNETIC ENVIRONMENTAL RISKS}

As it was mentioned before, environmental risks induced by electromagnetic radiation in wireless networks are determined by the aggregate level of radiation at the receptor location. As was shown above, the random part of this is dominated by the closest transmitter. Given a threshold value $P_{t h}$ (e.g. the MPL below), there is always a probability that the random power $P$ (see (1)) exceeds this level, which is the outage probability, $\mathcal{P}_{\text {out }}=\operatorname{Pr}\left\{P>P_{\text {th }}\right\}$.

There is a significant variation in maximum permissible levels (MPL) of electromagnetic exposure used in different countries and even different cities within the same country (and, of course, within different frequency bands). Some typical MPLs $\Pi_{E I}-\Pi_{E 4}$ in terms of the power density $\left(\mu \mathrm{W} / \mathrm{cm}^{2}\right)$ that are used as an electromagnetic safety criteria are shown below [5][9].

Table 1: Typical Maximum Permissible Levels

\begin{tabular}{|c|l|}
\hline$\mu \mathrm{W} / \mathrm{cm}^{2}$ & \multicolumn{1}{|c|}{ Description } \\
\hline 0.1 & $\begin{array}{l}\text { MPL for «total electromagnetic radiation exposure } \\
\text { from all high-frequency equipment with very low } \\
\text { pulsing modulation». Equal or close to the MPL } \\
\text { accepted in some countries/regions. }\end{array}$ \\
\hline 1.0 & $\begin{array}{l}\text { Level of electromagnetic background considered to } \\
\text { be safe for the general public as accepted in the } \\
\text { USSR up to 1984. }\end{array}$ \\
$\left(\Pi_{E 2}\right)$ \\
\hline 2.0 & $\begin{array}{l}\text { The MPL accepted in Moscow and Paris for places of } \\
\text { round-the-clock stay of people. }\end{array}$ \\
\hline $\left.1 \Pi_{E 3}\right)$ & $\begin{array}{l}\text { The MPL for the general public, accepted in Russia, } \\
\text { Belarus and also in a number of the European and } \\
\text { Asian countries (or close to it). }\end{array}$ \\
\hline
\end{tabular}

The power density level $\Pi_{b}$ of the receiver desensitization/blocking, determined at the antenna location, exceeds the receiver sensitivity $\Pi_{\min }$ via the blocking dynamic range $D_{d f b}$ :

$$
b=D_{d f b} \quad \min .
$$

One way for an outage to occur is when the actual interference level exceeds $b$. A comparison between the levels of radio receiver desensitization/blocking and the levels in the electromagnetic safety criteria, for typical values of receiver sensitivity $\Pi_{\min }$ and the receiver dynamic range $D_{d f b}$, are given in Table 2 .

Table 2 Comparison of levels

\begin{tabular}{|c|c|c|c|}
\hline$\Pi_{\min }$, & \multicolumn{3}{|c|}{$\Pi_{b}, \mu \mathrm{W} / \mathrm{cm}^{2}$} \\
\cline { 2 - 4 } $\mathrm{W} / \mathrm{m}^{2}$ & $D_{d f b}=70 \mathrm{~dB}$ & $D_{d f b}=80 \mathrm{~dB}$ & $D_{d f b}=90 \mathrm{~dB}$ \\
\hline $10^{-12}$ & $10^{-3}$ & $10^{-2}$ & $\Pi_{E 1}$ \\
\hline $10^{-11}$ & $10^{-2}$ & $\Pi_{E 1}$ & $\Pi_{E}, \approx \Pi_{E 3}$ \\
\hline $10^{-10}$ & $\Pi_{E 1}$ & $\Pi_{E 2} \approx \Pi_{E 3}$ & $\Pi_{E 4}$ \\
\hline
\end{tabular}

Clearly, the levels of out-of-band interference causing desensitization for highly-linear receivers (high $D_{d f b}$ ) roughly equal to the MPL levels established from the ecological point of view, which is an indication of close similarity between harmful interference and dangerous radiation exposure effects in wireless communication networks.

\section{REFERENCES}

[1] E.S. Sousa, Performance of a Spread Spectrum Packet Radio Network Link in a Poisson Field of Interferers, IEEE Trans. Inform. Theory, v.38, No.6, pp.1743-1754, Nov. 1992

[2] J. Ilow, D. Hatzinakos, Analytic Alpha-Stable Noise Modeling in a Poisson Field of Interferers or Scatterers, IEEE Trans. Signal Processing, v.46, No.6, pp.1601-1611, June 1998.

[3] G. L. Stuber, Principles of Mobile Communication (2nd Ed.), Kluwer, Boston, 2001.

[4] V.I. Mordachev, Typical Models of Electromagnetic Environments for Spatially-Scattered Radio Transmitters, in Proc. of the 10-th Wroclaw Symp. on EMC, June 1990, pp.409-414. (in Russian)

[5] V.Mordachev. System Ecology of Cellular Communications, Belarus State University Publishers, Minsk, Belarus, 2009 (in Russian).

[6] V.Mordachev, S.Loyka. On Node Density - Outage Probability Tradeoff in Wireless Networks, IEEE Journal on Selected Areas in Communications (Special Issue on Stochastic Geometry and Random Graphs for the Analysis and Design of Wireless Networks), v.27, No.7, p.1120-1131, Sept. 2009.

[7] J.C. Lin, Safety Standards for Human Exposure to Radio Frequency Radiation and Their Biological Rationale, IEEE Microwave Magazine, Dec. 2003, pp. 22-26.

[8] A.M. Martinez-Gonzalez et al, Practical procedure for verification of compliance of digital mobile radio base stations to limitations of exposure of the general public to electromagnetic fields, IEE Proc.-Microw. Antennas Propag., v. 149, N. 4, pp. 218 228, Aug. 2002.

[9] W. Van Loock, Basic Effects in Humans Exposed to Electromagnetic Energy, Occurrence of Cancer and Protection,VIII Int. Symp. on Electromagnetic Compatibility and Ecology, St. Peterrsburg, Russia, June 2009.

[10] "Estimation of Health Risks Induced by Cell Phone Radiation Based on Experimental Data", A Panel Discussion at the Russian Academy of Medical Sciences, Moscow, Russia, June 2010. 